\title{
Belphégor
}

\section{Passe, page, passage}

Notes sur « Le Passage à l'écran, littératures des hybrides »

\section{Paul Bleton}

\section{(2) OpenEdition}

\section{Journals}

Electronic version

URL: https://journals.openedition.org/belphegor/1322

DOI: $10.4000 /$ belphegor. 1322

ISSN: 1499-7185

Publisher

LPCM

Electronic reference

Paul Bleton, "Passe, page, passage”, Belphégor [Online], 16-1 | 2018, Online since 16 July 2018,

connection on 19 October 2021. URL: http://journals.openedition.org/belphegor/1322 ; DOI: https:// doi.org/10.4000/belphegor.1322

This text was automatically generated on 19 October 2021 .

\section{(c)}

Belphégor est mis à disposition selon les termes de la Licence Creative Commons Attribution - Pas d'Utilisation Commerciale - Pas de Modification 4.0 International. 


\section{Passe, page, passage}

Notes sur « Le Passage à l'écran, littératures des hybrides »

\section{Paul Bleton}

\section{Passages}

1 Dans une brique. C'est là que je relis «Le passage à l'écran. Littérature des hybrides ", dans les actes du colloque De l'écrit à l'écran: 870 pages! Matériellement, une fois empoignée cette compacité, la brique impose au lecteur l'emblème de l'établissement ; dans une double acception. Établissement car ce volume (le nom est mérité) a servi de première pierre, et même de fondations à la fédération puis à l'institutionnalisation d'un regroupement de chercheurs depuis logés à l'enseigne Littérature populaire et culture médiatique. Établissement aussi dans un sens plus défricheur de repérage et de reconnaissance d'un domaine de recherche.

Domaine de recherche? Il s'agissait en effet non plus simplement de chercher à collecter tout un pan de la production romanesque au bénéfice du catalogue encyclopédique in-progress ou de la bibliothèque virtuelle d'amateurs formant un cercle informel; non plus simplement de chercher à en obtenir la réhabilitation, au nom de l'adjectif populaire, lui-même ambigument entendu comme marqueur de classe ou comme marqueur de succès commercial, cependant que ce sous-ensemble de fictions était ignoré, voire méprisé par les études littéraires d'alors. Il s'agissait plutôt d'opérer une sorte de révolution copernicienne par rapport au réflexe rhétorique de légitimation qui avait cours. "Non, le roman populaire, ce n'est pas de la littérature mais en moins bien, en moins littéraire, en moins distingué, ce n'est pas le rez-dechaussée voire la cave de l'institution littéraire. » Le roman populaire, c'est plutôt la manière dont la culture médiatique s'est appropriée la fiction romanesque à ses propres fins, depuis le roman-feuilleton jusqu'à l'émergence de la cyberculture dont, en 1998, on pressentait la puissance reconfiguratrice.

3 Mais revenons à ce " Passage à l'écran » et à sa lecture. Livre en main, celle-ci doit aller au-delà de l'indubitable matérialité de la brique, au-delà de sa compacité, pour se rebrancher sur le vigoureux dynamisme qui animait les participants de ce colloque. 
Pour le regard rétrospectif de ceux qui ont poursuivi dans la voie indiquée par ce livre et qui en reprendraient la lecture (ce qui est mon cas), l'élan fondateur qui informait tout le projet affleure dans le texte de Charles Grivel, tout à fait directement, sans filtre, sans rides non plus.

Pourquoi ? Parce que ce texte démontrait le mouvement en marchant. Il affichait la fidélité de Charles au programme de défense du roman populaire. Qu'on imagine sa jubilation à dénicher l'article de F. de Lagevenais «La littérature illustrée » dans la Revue des deux mondes de Février $1843^{1}$, à en décortiquer la rhétorique à la fois consolatrice et furibonde - d'une part, malgré ses revers militaires, la France est grande par les œuvres de l'esprit, d'autre part, à cause de l'illustration qui s'insinue, s'infiltre, s'ingère dans les livres qui sont censés en être les tabernacles, l'admirable bâtisse est vermoulue, ses poutres bouffée par les larves de capricornes des maisons. Qu'on imagine enfin la jubilation de Charles à fournir à la perle servant de clausule à cette «Littérature illustrée » («l'illustration est un symptôme de décadence littéraire ») l'écrin d'ironie acide qu'elle méritait.

Mais surtout il montrait comment dépasser la gnoséologie d'érudition qui servait de dénominateur commun aux membres de l'Association des amis du roman populaire d'où Littérature populaire et culture médiatique procédait par méiose ${ }^{2}$. La recherche chez Charles se fondait bien sur une telle érudition, nécessaire et chez lui impeccable et gourmande; mais elle allait au-delà du seul empirisme descriptif. Il le recadrait, le soumettait à une problématique générale, proposait une démarche de savoir aporétique, risquée, exemplifiant le choix d'un passage ténu, aux antipodes de celui d'un globalisme totalisateur, passage ténu dont, quelques années plus tôt, un épistémologue de la communication avait montré la multiformité, la fécondité, la nécessité - Michel Serres et son Passage du Nord-Ouest (1980)³.

6 Passage, donc, comme démarche intellectuelle allant de l'érudition à la problématisation, de la visée encyclopédique à la réflexion théorique. Mais aussi passage comme cadrage-clé pour comprendre dans leur dynamisme les deux notions de base en études paralittéraires et en études médiatiques dont nous héritions, les genres et les médias, catégories pourtant spontanément ancrées dans la stabilité et la persistance, inoxydables, immarcescibles, inaltérables presque. En quoi d'ailleurs cette seconde acception de passage allait parfaitement illustrer le sous-titre du colloque qui annonçait "mutations génériques, mutations médiatiques", l'illustrer en toute érudition, en toute problématisation spéculative.

\section{Passe}

7 La curiosité de Charles, elle, n'était pas de passage ; elle émanait plutôt d'un intérêt ancien, continu et fertile de ce littéraire pour les différentes formes de coexistence de l'image et de la fiction narrative. Et pas seulement à partir du thème de l'adaptation qui ne lui était toutefois pas étranger, comme devait ultérieurement en témoigner sa participation au collectif de Jan Baetens et Marc Lits, La Novellisation: du film au roman (2004). Dès 1984, dans un article paru dans Lendemains, il s'intéressait à la photographie et, dès 1988, à ses croisements avec la littérature dans un numéro thématique «Photolittérature » qu'il avait monté avec Hubertus von Amelunxen pour le Revue des Sciences Humaines. Sans pouvoir tout mentionner, disons qu'à cette époque, du côté de l'illustration, il avait notamment étudié celles accompagnant Émile Zola, s'était mis au 
service de la redécouverte de Rodolphe Töpffer; du côté du cinéma, il avait retrouvé son grand Alexandre Dumas; il n'avait pas non plus hésité à visiter ses voisins philosophiques ${ }^{4}$. Ce qui allait s'avérer son intérêt de prédilection, la photographie, en était encore à l'adolescence - avec une brève contribution à La Recherche photographique et un article plus substantiel dans le volume 2 de Médusa-Médias, la revue de Mannheim, sur un thème familier, Photographie-Littérature. Cela dit, Charles n'avait cependant encore proposé aucune synthèse, ni même aucun belvédère conjectural d'où saisir dans leur ampleur les questions soulevées par la page imprimée en tant que sémiotope, en tant que lieu de convergence de mots et d'images. En revanche, par la proximité entretenue par lui avec la recherche en communication à l'université de Mannheim, notamment avec celle de Jürgen E. Müller qui était alors en train de découvrir dans l'intermédialité non pas un effet de la médialité mais son moteur même ${ }^{5}$, incubait alors chez Charles la réflexion qui allait mener à ce moment crucial dans sa carrière intellectuelle : les avancées de son « Passage à l'écran ». Moment crucial, passe étroite.

Après ce texte déterminant, Charles se mettrait à étudier les illustrations de couverture ainsi que le cinéma et plus généralement l'image, la vue et la vision, souvent associées au crime - singulièrement, pour les premières, lors de sa participation à un autre colloque de Limoges, Production(s) du populaire (2004), pour le deuxième, par exemple dans un numéro thématique "Dire l'indicible. Une écriture moderne de la vision " d'Études littéraires ${ }^{6}$, et pour les dernières comme dans «Troppmann ou de la défiguration" (2002) ou "Kip optogramme » (2005). Cela dit, c'est quand même la photo qui devait le plus le solliciter; pour ce qui nous concerne directement ici, il a poursuivi la piste de l'intermédialité ${ }^{7}$ et celle du cousinage du réalisme romanesque naturaliste avec la photo (Émile Zola, les frères Goncourt, Léon Bloy) ${ }^{8}$. Mais le crabe qui avait décidé de prendre en main sa carrière ne lui aura pas laissé le loisir de transformer le moment crucial de son « Passage à l'écran » en un grand œuvre.

\section{Sa page}

Prédisposition pour l'intermédialité ${ }^{\text {à }}$ la Müller? Toujours est-il que Charles, ce biculturel cosmopolite et polyglotte, attiré aussi bien par le nomadisme avide d'ailleurs et d'autrui que par l'austère sédentarité de l'écriture, éprouvait une affinité, colorée par un navrement personnel, pour le mélange et pour la complexité.

10 «L'image a pénétré le texte. » (p. 407) : voilà qui aurait enclenché l'investigation sur un état de l'imprimé vers 1850. L'affaire était pourtant bien plus ancienne puisque le premier incunable réussissant à intégrer texte (latin et allemand) et images avait paru à Nuremberg chez Anton Koberger en 1493 ! Ce n'est pas parce qu'elle n'était pas une fiction narrative que Charles n'en parle pas - quoiqu'intitulée Chronique de Nuremberg, cette chronique universelle de Hartmann Schedel (traduite par Georg Alt) s'avère largement fictive. C'est plutôt parce que Charles s'intéresse à la littérature populaire, à la fiction romanesque en régime médiatique. Telle est aussi la raison qui lui fait éviter la causalité technologique unique de la médiologie, sans doute trop simplificatrice: certes Charles ne néglige pas dans l'équation les progrès technologiques (lithographie, gravure de bois de bout, puis photo, similigravure et typogravure...), mais il ne déduit ni une philosophie de la création ni une esthétique d'une innovation technique.

11 Son expérience de lecteur et de collectionneur lui fournit une première intuition, celle d'une forte parenté entre des produits imprimés apparemment disparates mais tous 
issus de la tendance lourde de la Révolution industrielle à élargir les marchés, y compris celui de la Littérature, pourtant domaine du qualitatif. Or, pour Charles, cette parenté est moins technique que sémiotique et culturelle : tous marient narration et figuration.

Seconde intuition, en fait si profondément grivellienne qu'elle constitue aussi une seconde nature pour tous ses textes : ces objets best-sellers prospèrent moins parce qu'ils seraient techniquement innovateurs, formellement réussis ou créativement inspirés que parce qu'ils captent un désir, ici un "désir d'images». Ce qui plante le décor pour le drame à venir, la dernière phase de sa réflexion, la compétition entre narration et figuration, entre l'objet de deux désirs (voir et lire), entre les deux composants du composite qu'il s'est donné à étudier.

Cette première phase arrime l'intuition à deux généralisations. La première : quelle que soit la définition de la page imprimée, elle doit choisir entre deux figures contemporaines, Johannes Gutenberg ou Leon Battista Alberti, c'est-à-dire la page alphabète de la typographie et du "pavé » bidimensionnel de l'imprimeur ou la pagefenêtre déterminant une topographie, l'emplacement de l'image à la fois dans l'espace de la page et dans le fil des phrases. La seconde : pendant que l'attention se tourne vers le vu et le lu, la matérialité de la surface imprimée en profite pour s'esbigner, pour disparaître de la conscience. Il faut donc redoubler l'attention portée à la page alphabète et à la page-fenêtre d'une attention portée aux supports, au format, au volume...

14 Ce qui conduit à la deuxième phase, érudite, celle qui permet dans un même geste d'affirmer la médiativité spécifique de son objet (la fiction imprimée ${ }^{10}$ et de desserrer la solidarisation qui nous semble pourtant spontanée entre la fiction romanesque et son support-livre. Si le roman populaire c'est la manière dont la culture médiatique s'est appropriée la fiction romanesque, faire l'histoire de l'édition populaire c'est accepter de changer de lorgnette ${ }^{11}$. Méthodologiquement, cela revient à distinguer les matériaux sémiotiques composites qu'annexe la fiction du support et de ses formats - c'est-à-dire le dispositif technique à travers lequel le lecteur voit et lit. Cela revient aussi à distinguer dispositif technique et matériaux sémiotiques de la diachronie des genres romanesques (ceux dont elle hérite, qu'elle mixe et qu'elle modifie), tout autant que de la synchronie des séries culturelles avec lesquelles la fiction romanesque entre en résonnance ${ }^{12}$.

Alors que malgré d'importantes mutations de l'industrie du livre alors en cours, une période d'institutionnalisation du roman populaire en avait stabilisé les formes, le regard rétrospectif de Charles sur la fiction populaire du XIX siècle lui fait mettre sous les projecteurs l'instabilité du mélange narration/figuration sur fond de stabilité relative de la médiativité de l'imprimé. Il en réalise la démonstration par la comparaison de quatre formats distincts - que, à juste titre, il présente comme autant d'échantillons puisque bien d'autres cocktails ont été effectivement tentés, alors et depuis, par les éditeurs de fiction populaire et leurs industries graphiques.

Pour des raisons justement liées au conservatisme technique obligé de la brique (qui n'a pu reproduire que deux cartes postales, savoureuses, certes, mais qui laissent le lecteur sur sa faim), le texte de Charles sollicite fortement la mémoire visuelle de son lecteur et ne prend toute sa signification que lorsque, sur une même table, ce lecteur peut se mettre sous les yeux des spécimens de romans populaires illustrés des années 1870-80, des revues de romans illustrés par gravures et photos, parfois en alternance, des séries 
narratives de cartes postales (le composite le plus éloigné de la forme-livre, un autre dispositif, sans doute) et les romans-cinéma des années 1920.

Dès lors, la troisième phase, sorte d'extension de la démarche narratologique au récit devenu hybride consiste à comprendre l'espace et le volume de la fiction populaire. Elle met à jour les figures des relations entre narration et figuration. Relations fonctionnelles (illustration d'une scène, d'un personnage), relations pragmatiques (augmenter ou diminuer la tension psychique $\mathrm{du}$ lecteur), relations temporelles (décalage ou simultanéité? décalage par retardement ou décalage par anticipation?), relations sémiotiques (redoublement d'une scène écrite, d'une prosopographie, ou substitution, l'image complémentant une narration restée muette) ? La dernière phase constitue elle aussi une seconde nature chez Charles, l'attention portée aux relations de pouvoir. Déroulant cette histoire de la coexistence de la narration et de la figuration, il finit par mettre en lumière une histoire d'adultère. Depuis l'apparition d'une Lesewut en Europe de l'Ouest, d'une rage de lire ${ }^{13}$ correspondant à la montée en puissance de cette formule paradoxale d'une lecture à la fois extensive par la multiplication des titres et intensive par l'intériorisation individuelle qu'elle exige, la narration semblait mariée à la langue imprimée. Ce qui irritait déjà les censeurs comme un double pervertissement : dévoiement de la religion puisque l'intensivité ne s'appliquait plus simplement à des ouvrages religieux mais à des romans aussi ${ }^{14}$ et dépravation de la parole comme fondement du lien social puisque l'intériorisation «désocialisait» le lecteur et désocialisait les pratiques de lecture antérieures ${ }^{15}$. Sans dire qu'elle affectait une proportion d'autant moins négligeable de la population qu'elle croissait sans cesse $^{16}$. Mais depuis que la narration imprimée s'était mise à flirter avec la figuration sous la même couverture, voilà que la langue imprimée se voyait moins aimée, prise pour acquise, redoutant d'être délaissée ou rétrogradée en position subalterne. Et si, en séquence, les images imaginaient narrer sans mots ? Et si le lu encré sur la page était désancré par les vues animées ? Le discours des censeurs était déjà tout prêt, bien rôdé, malgré la nouveauté des technologies mises au service de la narration fictionnelle.

\section{Page-écran}

Non, il n'y a pas à s'emballer là pour quelque anticipation vernienne de la tablette tactile dans le texte de Charles. Si l'on doit s'enthousiasmer pour sa page, cet hybride non pas technologique mais conceptuel de la page-écran, c'est plutôt à cause de la puissance d'une troisième intuition, d'une grande idée issue d'un imaginaire à la fois visionnaire et spéculatif - une allégorie spontanée, un emblème phénoménologique, le renversement de ce qu'était en train de révéler l'écran d'ordinateur, lui-même devenu tout à la fois écran-page et écran-fenêtre sur lequel s'étaient croisés texture du texte et canevas de la toile (le jupon ne dépassait-il pas lorsque l'informatique s'était penchée sur les fonds baptismaux et avait nommé Word et Windows ?) Lors de sa patiente analyse de ces formats expérimentés par l'industrie paralittéraire, quelle représentation avait donc foudroyé Charles par son évidence sensible?

D'un côté, il y avait la chrono-logique des choses : en amont, le souvenir de la page alphabète des livres dont il restait un avide lecteur; sous les yeux, la diversité de l'investissement de la surface imprimable par des cocktails de mots et d'images; et en aval, alors? Dans son bouquet de formats, le roman-cinéma était la pointe extrême 
avant le basculement, avant que la figuration ne prenne en charge la narration, en ne laissant au mot qu'une portion congrue (comme en BD, avec légendes et phylactères) ou en désolidarisant les temps et les espaces de la réception (comme temps et espace du film muet et temps et espace de son roman-cinéma). En aval, donc: la narration par images, sans mots peut-être, l'hégémonie de la figuration. Placé plus en aval encore, Charles pouvait distinguer la logique d'un enchaînement qui faisait de la fiction romanesque un moment de l'histoire de la culture filmique, du livre un moment de l'histoire du cinéma...

D'un autre côté, il y avait la poétique des mots : de ces mots comme le sacer latin, le deep anglais, l'Aufhebung allemand dont la dialectique hegelienne avait fait son moteur (suppression et élévation, relève, disait Jacques Derrida) qui incitent à la rêverie spéculative parce que, à double face, ils disent une chose et son contraire. Pour le texte de Charles, l'écran français. Et voilà que, comme une sorte de fractale, la conception du livre comme moment de l'histoire du cinéma se reproduit dans ses pages, chacune s'avère un écran.

Depuis le cinéma, l'écran est ce sur quoi se projette la vue animée, ce qui capte le regard et permet au spectateur de se projeter dans ce qu'il voit. Comme l'imprimé sur la page est toujours déjà-là pour le lecteur, contrairement à la vue animée sitôt projetée sitôt consommée, la page-écran ne serait-elle que cette seconde moitié du dispositif? Pas sûr. En un autre sens, plus primitif, l'écran empêche de voir ; les mots imprimés sur la page gutenbergienne s'interposent entre le regard du lecteur et l'univers fictionnel auquel il désirerait tant accéder; elles s'interposent mais en même temps elles sont le seul moyen d'y accéder. C'est l'image imprimée sur la page albertienne qui en fait prendre conscience. Par ailleurs, le penchant de Charles pour le paradoxe ne saurait négliger de souligner la capacité d'énigmatisation, voire d'aveuglement de l'image: parmi toutes les significations possibles, son sens actuel, avéré, ce sont les phrases du récit qui le lui attribue.

Telle est l'intuition de Charles : sa page est un écran, et toute l'ambiguïté qui vient avec. En matière de lecture, certes, à la fois page qui masque et page qui révèle, page qui révèle le monde et, se faisant, révèle le lecteur séduit à lui-même. Mais aussi en matière d'études médiatiques, lorsqu'on file la chronologie des relations sous une même couverture de la narration et de la figuration, la page est tout autant l'emblème de l'impasse, de la clôture de l'écrit imprimé voire de la culture littéraire que l'emblème de la passe, étroite ou pas. C'est en passant par toutes ces hybridations sémiotiques narration/figuration que Charles dessine une théorie de l'évolution de la fiction populaire faite de continuité et de mutations de ses espèces, du livre au film. Devenue écran, sa page s'avère passage.

Reste aujourd'hui un écran, partagé entre ce que vous y lisez et ce que j'y projette. Vous y lisez le balancement de l'écriture de Charles, entre ses langues, entre ses styles, de la raideur de listes de thèses à la Althusser dans Production de l'intérêt romanesque jusqu'aux rhapsodies phénoménologiques d'articles plus tardifs. On pressent dans un tel le balancement l'affinité spontanée avec le thème traité par le texte, le mélange, les formes que peut prendre l'hybridation, ce que la "métaphysique des mélanges " d'Emanuele Coccia (2016) propose de nommer une "autotrophie spéculative». Incidemment, cet intérêt pour la "littérature des hybrides » met en branle une démarche discrète certes, limitée à l'objet de la recherche mais qui n'en a pas moins une plus large portée. Elle s'éloigne en effet, en toute quiétude, du lourd héritage du 
pur et de l'impur qui empêtrait aussi bien la réflexion sur la littérature qu'elle surdéterminait celle sur le paysage culturel européen ${ }^{17}$.

En relisant ce « Passage à l'écran », je constate, et vous le pouvez aussi, que ce colloque d'il y a 20 ans (il s'est tenu en 1998) a parfaitement correspondu aux attentes du petit groupe qui, suite à plusieurs échanges déjà internationaux mais de format plus modeste, en était venu à croire à la nécessité de monter en puissance, de rencontrer, fédérer et institutionnaliser les chercheurs universitaires intéressés aux problématiques de la confluence de la fiction romanesque et de la culture médiatique, travaillant en solo et en chœur mais tous un peu en silo dans leurs milieux de recherche nationaux. Toutefois, cette montée en puissance n'aurait su se réduire à un accroissement du nombre d'adhésions. Elle vient plutôt de ce que des Charles Grivel ont intégré dans leurs propres problématiques de recherches les tensions que provoque la cohabitation de la fiction romanesque et de la culture médiatique, ont nourri leurs réflexions des compétitions issus de cette promiscuité.

En relisant ce "Passage à l'écran ", sur le mien s'affichent les souvenirs. Souvenirs du chaleureux: tumultueux, attentif, séducteur, la bouche large, l'articulation tendue d'une voix faite pour expliquer, pour rendre évident, pour s'enquérir ou la nasalisation d'une parole de grommellement ou de confidence sotto voce... Mais aussi souvenirs de l'infatué de paradoxes, les yeux pétillants derrière des lunettes cerclées. J'entrevois moins dans ses propres paradoxes le goût pour eux du maître zen que la ruse du dialecticien. Le premier incite à lâcher prise alors que le second (chez Charles déguisé en un dramaturge) prépare soigneusement sa surprise pour faire exulter la réflexion de son lecteur. Comment ne pas jubiler devant «[...] l'image figure un seuil inconscient, une latence, une énigme: sa présence pour "authentique» qu'elle soit, irradie l'inquiétude. De telle sorte que plus l'exactitude de la représentation paraît assurée, plus augmente aussi son illisibilité : une image en dit trop par essence ou n'en dit pas assez. » (p. 410) ?...

En relisant ce « Passage à l'écran », il faut bien se rendre à l'évidence, Charles est passé. Certes ; mais juste de l'autre côté du miroir.

\section{BIBLIOGRAPHY}

ANGENOT, Marc. 1889. Un état du discours social, Longueuil, Le Préambule, coll. L'univers du discours, 1989.

CocciA, Emanuele. La Vie des plantes. Une métaphysique du mélange, Paris, Payot \& Rivages, coll. Bibliothèque Rivages, 2016.

DEBRAY, Régis. Cours de médiologie générale, Paris, Gallimard, coll. Bibliothèque des idées, 1991. GRIVEL, Charles. « Au bout de la vue. Félix Nadar, Paul Nadar, Étienne Carjat, Sarah Bernhardt », Lendemains, $\mathrm{n}^{\circ} 34,1984$, p. 49-63. 
GRIVEL, Charles. « Kip optogramme. Le Roman du crime et de la vue ». Jules Verne cent ans après. Actes du colloque de Cerisy. Jean-Pierre Picot et Christian Robin (sous la dir. de), Rennes, Terre de brume, 2005, p. 63-85.

GRIVEL, Charles. « Photocinématographication de l'écrit romanesque », La Novellisation: du film au roman/ Novelisation : From Film to Novel, Jan Baetens \& Marc Lits (eds) Leuven University Press, 2004, p. 21-39.

GRIVEL, Charles. « De la couverture illustrée du roman populaire », Production(s) du populaire.

Colloque international de Limoges (14-16 mai 2002), Jacques Migozzi \& Philippe Le Guern (réunis par), Limoges, PULIM, 2004, p. 281-305.

GRIVEL, Charles. « Un œil en trop. Les Goncourt photographiques », Cahiers Edmond et Jules de Goncourt, $\mathrm{n}^{\circ} 11,2004$, p. 103-120.

GRIVEL, Charles. « Zola, photogénèse de l'œuvre », Études photographiques, n 15, novembre 2004, p. 31-60.

GRIVEL, Charles. «Troppmann ou de la défiguration », Eine Literatur für den Leser, Jörg Türschmann (éd.), Aix-la-Chapelle, Shaker Verlag, coll. Médusa Médias, n 5, 2002, p. 73-104.

GRIVEL, Charles. « Photographie, littérature, peinture, cinéma : la guerre des arts », Intermediale: kommunikative Konstellationen zwischen Medien, Beate Ochsner \& Charles Grivel (Hrsg.), Tübingen, Stauffenburg Verlag, 2001, p. 11-44.

GRIVEL, Charles. « Dieu photographe. Bloy, ou l'Absolu de la photographie », Léon Bloy n 5 : Léon Bloy et la communication dans «l'enfer des médias ", Pascal Durand (textes réunis et présentés par), Paris-Caen, Lettres Modernes Minard, 2001.

GRIVEL, Charles. «Le passage à l'écran. Littérature des hybrides », Jacques Migozzi (sous la dir. de) De l'écrit à l'écran, Limoges, PULIM, 2000, p. 407-435.

GRIVEL, Charles. «Le roman mis à nu par la photographie, même », L'imaginaire photographique, Romantisme, $\mathrm{n}^{\circ}$ 105, 1999, p. 145-162.

GRIVEL, Charles. Rodolphe Töpffer, réflexions et menus propos d'un peintre genevois ou Essai sur le beau dans les art, suivi de De la plaque Daguerre, préface, chronologie et bibliographie, Paris, Ecole Nationale Supérieure des Beaux-Arts, coll. Beaux-Arts Histoire, 1998.

GRIVEL, Charles. « Le voir. Réflexion de la photographie », Photographie-Littérature. Médusa-Médias, 2, Alain Buisine et Emmanuel Watteau (éds), 1995, p. 1-26.

GRIVEL, Charles. « Le portrait de la photographie », La Recherche photographique, $\mathrm{n}^{\circ} 14$, printemps 1993, p. 24-27.

GRIVEL, Charles. « Zola. Comment voit-on ? Illustration, non illustration », Les Cahiers naturalistes, $\mathrm{n}^{\circ}$ 66, 1992.

GRIVEL, Charles. « Hublots. La visée sur le vif », M/I/S. Mots/Images/Sons. Actes du colloque international de Rouen, 14-17 mars 1989, CIREM/Siph, 1990, p. 193-208.

GRIVEL, Charles. « La couverture d'images », Texte et médialité, Jürgen E. Müller (éd.), Mannheim, MANA 7, 1987, p. 127-189.

GRIVEL, Charles et LEBLANC, Gérard. L'aventure-cinéma et Alexandre Dumas, Ville de Villers-Cotterêts/ Limonaire, 1994. 
GRIVEL, Charles. et Hubertus v. AMELUNXEN. Photolittérature. Revue des Sciences Humaines, $\mathrm{n}^{\circ} 210$, 1988.

HIGGINS, Dick. The Intermedia Essay, The Something Else Newsletter, vol. I, n 1, New York, Something Else Press Ed. 1966.

KÖNIG, Dominik v. « Lesesucht und Lesewut », Buch und Leser, Herbert G. Göpfert (ed.), Hamburg, Hauswedell, 1977, p. 89-124.

LAGEVENAIS, F. de. « La littérature illustrée », Revue des deux mondes, février 1843 http://

rddm.revuedesdeuxmondes.fr/archive/article.php?code=70494

MARION, Philippe. « Narratologie médiatique et médiagénie des récits », Recherches en

communication, $\mathrm{n}^{\circ}$ 7, 1997, p. 61-87.

PARENT, Françoise. « De nouvelles pratiques de lecture », Histoire de l'édition française, t. 2, Le livre

triomphant, 1660-1830, sous la dir. de Roger Chartier et Henri-Jean Martin, 1990, p. 801-818.

SCARPETTA, Guy. Éloge du cosmopolitisme, Paris, B. Grasset, coll. Figures, 1981.

SERRES, Michel. Le Passage du Nord-Ouest. Hermès 5, Paris, Minuit, coll. Critique, 1980.

THIESSE, Anne-Marie. Le Roman du quotidien : lecteurs et lectures populaires à la Belle-Époque, Paris, Seuil, collection Points, $n^{\circ}$ 277, 2000 [1984].

\section{NOTES}

1. Aujourd'hui accessible directement sur le web, il fallait fouiller plus longtemps à cette époque pour parvenir à lui.

2. Faut-il rappeler que Charles présida cette association six ans durant, de 1993 à 1999.

3. Lui suggérait le modèle de la navigation dans ces eaux imprévisibles pour réussir de fructueux échanges entre sciences humaines et sciences exactes et vice versa.

4. Comme avec « Hublots. La visée sur le vif» (1990), dans les actes d'un colloque international organisé conjointement à Rouen en 1989 par le Centre international de recherche en esthétique musicale et le Collège international de philosophie et réunis dans un $n^{\circ}$ spécial de $M / I / S$. Mots/ Images/Sons.

5. Voir notamment l'article de Charles «La couverture d'images » (1987), paru dans un collectif sous la direction de Müller dans la collection MANA (Mannheimer Analytika) publiée par la Lehrstuhl Romanistik de cette université.

6. Une revue qui paraît à Québec.

7. Comme dans «Photographie, littérature, peinture, cinéma : la guerre des arts» (2001), paru dans un collectif qu'il avait monté avec Beate Ochsner.

8. On ne pourra pas aborder ici ce que Charles a pensé dans la demi-douzaine d'articles consacrés à l'imaginaire proprement photographique.

9. Écho d'une notion proposée par Dick Higgins, éditeur de Something Else Press et infatigable expérimentateur artistique, dans The Intermedia Essay (1966).

10. C'est-à-dire, pour Philippe Marion (1997), le potentiel spécifique d'un média, sa capacité propre à représenter.

11. D'élargir par exemple imprimerie à industries graphiques - même si cette expression, émergée dans les années 1950, est anachronique pour la période envisagée.

12. Qu'on pense notamment à la série du romanesque-général, cette forme cognitive privilégiée par le discours social pour comprendre et représenter le monde, notion proposée par Marc Angenot (1989). 
13. Selon Dominik v. König (1977), l'expression daterait de 1773 (dans les Briefe über die Erziehung der Frauenzimmer de Rudolph Heinrich Zobel). Elle s'est toutefois imposée dans l'espace social l'année suivante avec la parution des Souffrances du jeune Werther.

14. Avant le roman de Johann Wolfgang von Goethe, le processus avait commencé avec ceux de Samuel Richardson et Jean-Jacques Rousseau...

15. Cf. Françoise Parent (1990).

16. Ainsi, Anne-Marie Thiesse (1984) rappelle que, avant même les lois Ferry, 66\% des conscrits savaient lire en $1851-1852,82 \%$ en $1871-1875 .$.

17. Sur ce dernier point, politique, qu'on se souvienne de la parution de l'Éloge du cosmopolitisme de Guy Scarpetta (1981).

INDEX

Mots-clés: Grivel, littérature populaire, culture médiatique

\section{AUTHOR}

\section{PAUL BLETON}

Téluq, Montréal 